\title{
Nodular swelling of the buccal mucosa $\underline{s}$
}

Luciana Yamamoto Almeida, DDS, PhD, ${ }^{\mathrm{a}}$ Darcy Fernandes, DDS, MSc, ${ }^{\mathrm{a}}$ Túlio Morandin Ferrisse, DDS, ${ }^{\mathrm{a}}$ Rose Mara Ortega, DDS, PhD, ${ }^{a}$ Daphine Caxias Travassos, DDS, ${ }^{a}$ Alfredo Ribeiro-Silva, MDS, PhD, ${ }^{b}$ Jorge Esquiche León, DDS, $\mathrm{PhD},{ }^{\mathrm{c}}$ and Andréia Bufalino, DDS, $\mathrm{PhD}^{\mathrm{a}}$

(Oral Surg Oral Med Oral Pathol Oral Radiol 2017;123:150-156)

\section{CLINICAL PRESENTATION}

A 78-year-old white woman was referred to the Oral Medicine Service of Araraquara Dental SchoolUNESP, Brazil, for diagnosis and management of a nonulcerated submucosal nodule on the left side of the buccal mucosa (Figure 1A). According to the patient, it has been present for about 1 week. Clinical examination revealed a fluctuant nodular submucosal mass and slightly symptomatic on palpation. The lesion measured approximately $2 \mathrm{~cm}$ in greatest dimension, and it was covered by normal-appearing mucosa. Regional lymphadenopathy was not detected. Her medical history was noncontributory, and the patient did not report fever, weight loss, malaise, and fatigue.

\section{DIFFERENTIAL DIAGNOSIS}

Considering the clinical presentation and localization of the current lesion, differential diagnoses were focused on benign neoplasms of mesenchymal or glandular origin, as well as reactive lesions. Furthermore, due to the rapid growth of the lesion, malignant neoplasms of salivary gland, lymphoid, and mesenchymal origin were also considered.

\section{Benign mesenchymal tumors}

In the current nonulcerated nodular lesion, the diagnosis of submucosal mesenchymal neoplasms of neural (schwannoma, neurofibroma), adipocytic (lipoma), fibroblastic (solitary fibrous tumor), smooth muscle (leiomyoma), and myofibroblastic (myofibroma and nodular fasciitis) origin should be considered.

\footnotetext{
This abstract was presented as a poster on July 07, 2015, at the 23rd Brazilian Congress of Oral Medicine and Pathology, held in São Paulo, Brazil.

${ }^{a}$ Department of Diagnosis and Surgery, Araraquara Dental School, Universidade Estadual Paulista (UNESP), Araraquara, São Paulo, Brazil.

${ }^{\mathrm{b}}$ Department of Pathology, Ribeirão Preto School of Medicine, University of São Paulo (USP), Ribeirão Preto, São Paulo, Brazil.

c Oral Pathology, Department of Stomatology, Public Oral Health, and Forensic Dentistry, School of Dentistry of Ribeirão Preto, University of São Paulo (USP), Ribeirão Preto, São Paulo, Brazil.

Received for publication Aug 9, 2015; returned for revision Dec 2, 2015; accepted for publication Feb 6, 2016.

(c) 2016 Elsevier Inc. All rights reserved.

2212-4403/\$ - see front matter

http://dx.doi.org/10.1016/j.00oo.2016.02.005
}

Schwannoma and neurofibroma are benign peripheral nerve sheath tumors. Intraoral involvement accounts for approximately $1 \%$ of these tumors located in the head and neck region. ${ }^{1,2}$ Between $20 \%$ and $60 \%$ of oral neurofibromas are associated with neurofibromatosis, being that approximately $3 \%$ to $15 \%$ of these cases present potential for sarcomatous transformation. Intraoral schwannomas and neurofibromas show a predilection for the tongue, followed by the buccal mucosa, palate, floor of the mouth and lip. ${ }^{1}$ Although they can arise at any age, the average incidence is between 20 and 50 years, being more common in females. ${ }^{2}$ These tumors manifest as an asymptomatic nodular swelling. ${ }^{1}$ Microscopically, schwannoma shows a well-defined and encapsulated tumor mass comprising Schwann cells, whereas neurofibroma is an ill-defined mass and composed of Schwann cells, perineural cells, and endoneural fibroblast-like cells. ${ }^{2}$

Lipoma is a benign mesenchymal neoplasm that comprises about $1 \%$ to $4 \%$ of all benign tumors of the oral cavity. ${ }^{3,4}$ Clinically, it appears as a low-growing, painless, well-defined mass yellow in color. An elastic consistency may be evident, depending on the amount of fibrous tissue. ${ }^{3}$ The sites more frequently involved are the buccal mucosa and tongue, especially in males over 40 years of age. ${ }^{3,4}$ Microscopically, lipomas show a well-circumscribed mass composed mainly of mature adipocyte separated by thin vascular fibrous septae. $^{3}$

Solitary fibrous tumor (SFT) is a spindle-cell neoplasm of fibroblastic origin and occurs most commonly in the pleura. ${ }^{5}$ To date, approximately 80 cases of oral SFT have been reported in the English language literature. The buccal mucosa is frequently affected, followed by the tongue, gingiva, palate, and lip. Oral SFT typically shows a low-growing asymptomatic submucosal mass of varying size, affects patients in their sixth decade of life, with equal gender predilection. ${ }^{5}$ Histopathologically, SFT shows densely cellular areas alternating with hypocellular areas in a collagenous stroma. The tumor cells show immunoreactivity for CD34, CD99, and bcl-2. ${ }^{5}$

Leiomyoma is a benign smooth muscle tumor, being commonly found in uterine myometrium (95\%), and less than $1 \%$ is detected in the head and neck region. Oral leiomyoma accounts for about $0.05 \%$, and clinically it appears as an asymptomatic submucosal mass, most 


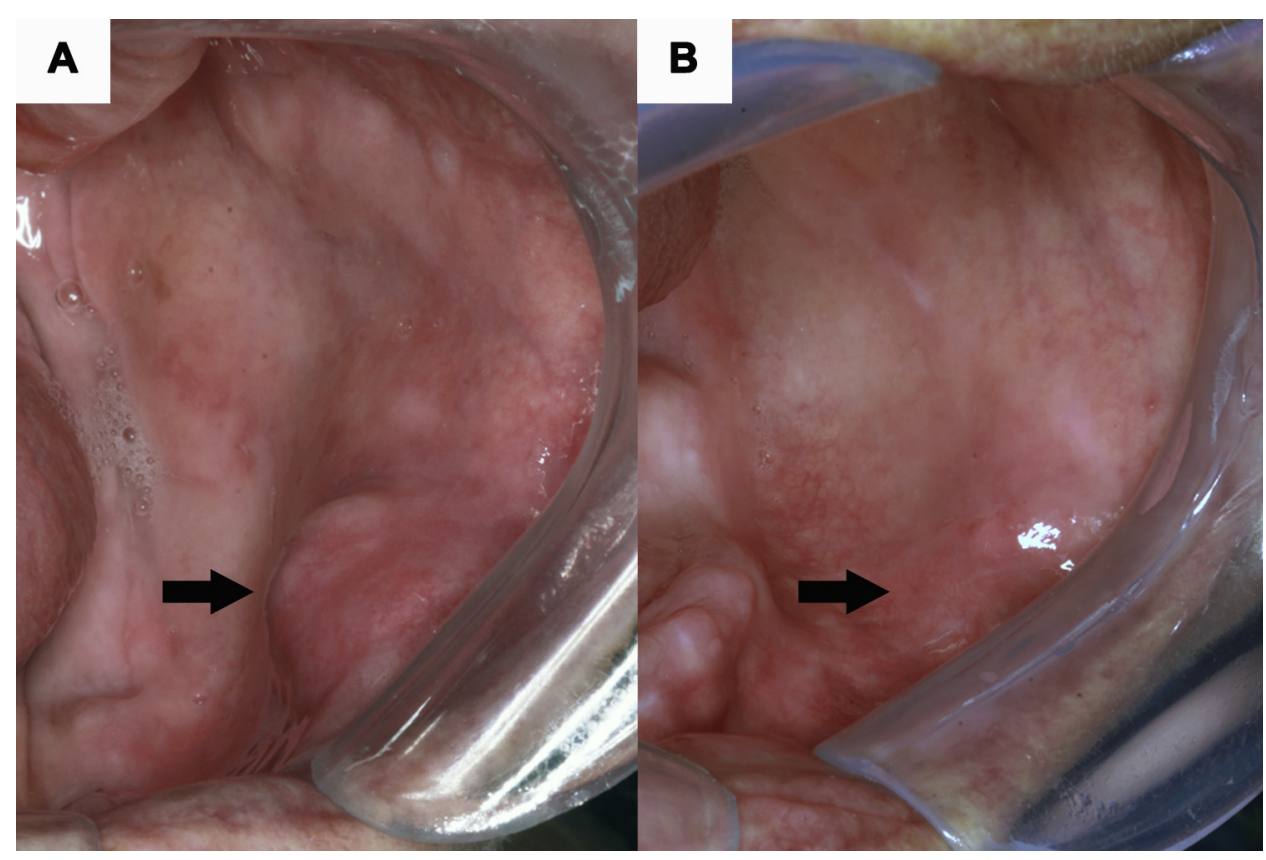

Fig. 1. Clinical aspects of the left buccal mucosa lesion diagnosed as subacute necrotizing sialadenitis (SANS). A, Submucosal non-ulcerated nodule (arrow). B, At 1-month follow-up, the lesion is completely healed (arrow).

frequently affecting the lips, followed by the tongue, buccal mucosa, palate, gingiva, and mandible. ${ }^{6,7}$ It has a slight male predilection and a peak incidence between 40 and 49 years of age. ${ }^{7}$ Angioleiomyoma is the most common variant affecting the oral cavity (64\% of all cases), followed by the solid and epithelioid variants. ${ }^{6}$ Histopathologically, consists of a circumscribed mass composed by spindle-shaped cells with eosinophilic cytoplasm and cigarlike shaped nuclei admixed with variable vascular stroma. ${ }^{6,7}$

Myofibromas are benign myofibroblastic tumors, with approximately $50 \%$ of them occurring in cutaneous tissues of head and neck region. ${ }^{8,9}$ Oral soft tissue myofibroma is relatively rare, with approximately 44 cases reported in the English language literature, frequently affecting the tongue (32\%), followed by the buccal mucosa $(18 \%)$. There is a slight predilection for the female gender (ratio 1:1.6), with age ranging from birth to 70 years (mean, 21.7 years). ${ }^{8}$ Clinically, a painless, firm, solitary swelling usually covered by normal mucosa is observed, and it may mimic other soft tissue tumors of neurogenic and smooth muscle origin. Besides, myofibroma can demonstrate a slow or even a rapid growth rate, making the diagnosis challenging. ${ }^{8,9}$ Microscopically, a spindle cell proliferation exhibits a biphasic pattern around irregular blood vessels (hemangiopericytic pattern). ${ }^{9}$ In difficult cases, immunohistochemistry, associated with careful morphologic analysis, is useful. Likewise, this is valid for all other tumors previously mentioned.
Nodular fasciitis (NF) is a benign myofibroblastic proliferation that frequently occurs in the subcutaneous tissues of the upper extremities, as well as head and neck region. NF occurs equally in both genders, predominantly between the ages of 20 and 40 years. ${ }^{10,11}$ Unfortunately, NF is not uncommonly misdiagnosed as sarcoma, owing to its rapid growth (few weeks), and microscopically by exhibiting high cellularity and high proliferative activity. To date, approximately 45 cases of oral NF have been reported in the English language literature, being the buccal mucosa the most common location. ${ }^{11}$

\section{Reactive lesions}

Despite having no history of trauma, a diagnosis of mucocele was also considered. Mucocele is a common reactive lesion of the oral mucosa, resulting from ductal obstruction, and typically shows a mass with translucent aspect, and it frequently affects the lower labial mucosa. ${ }^{12}$ Microscopically, the mucocele exhibits mucus extravasation surrounded by a wall of granulation tissue. ${ }^{12}$ Fibrous hyperplasia $(\mathrm{FH})$ is the most frequent reactive lesion of the oral cavity, and it clinically appears as a pedunculated or sessile growth as a result of trauma or local irritation. ${ }^{13}$ FH can be found in the buccal mucosa along the bite plane, as it was described in the current case. Microscopically, FH is characterized by large collagen fibers deposition. ${ }^{13}$ In the current case, these reactive lesions were considered as less probable diagnoses. 


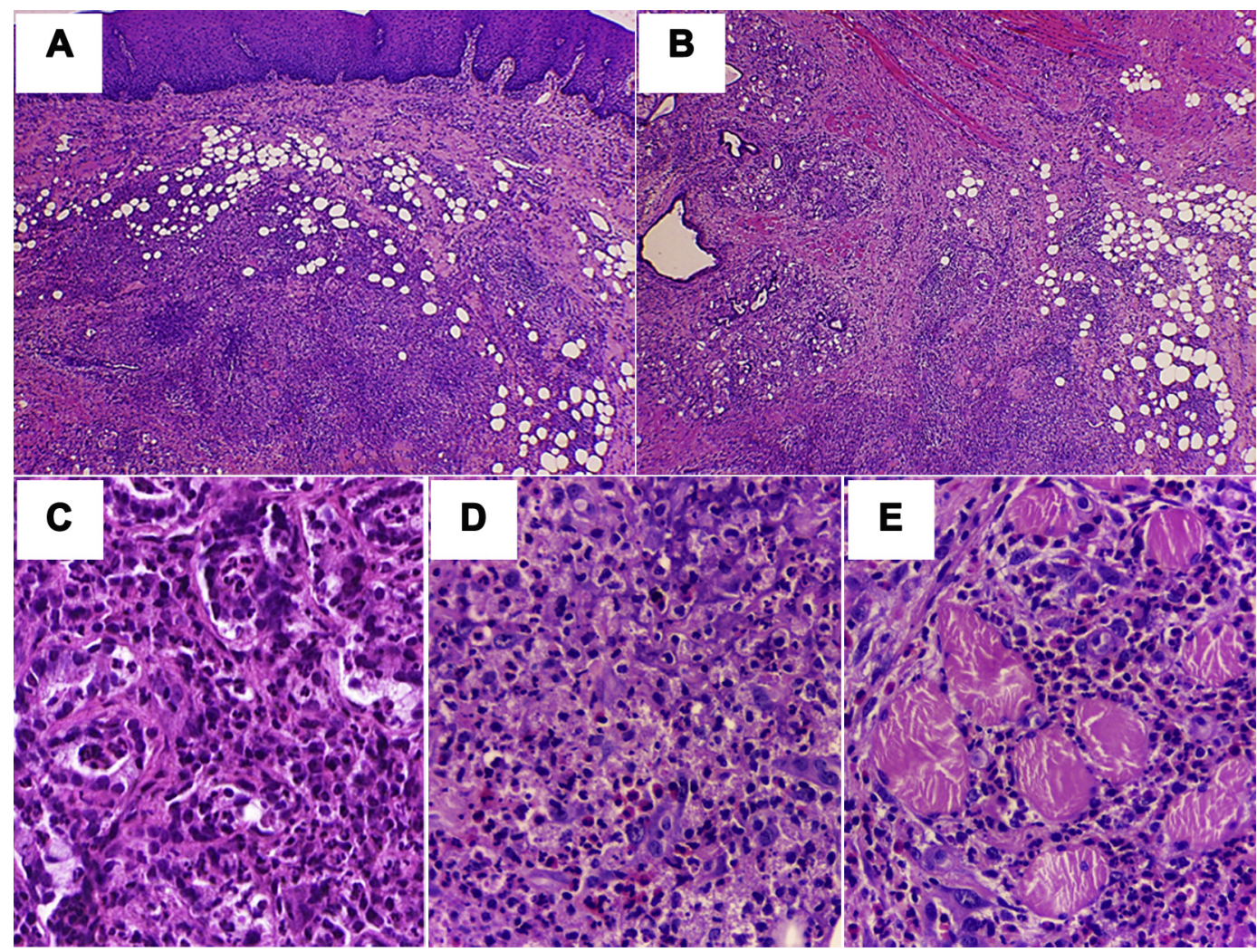

Fig. 2. Histopathological features. A, Intact surface epithelium associated with a submucosal exuberant inflammation. B, At a deeper level, note the presence of inflammatory cells diffusely infiltrating minor salivary glands, bundles of skeletal muscle fibers, and adipose tissue. C, Close-up view showing parenchyma of minor salivary glands with areas of necrosis. D, Besides eosinophils, neutrophils, and plasma cells, macrophages are abundantly present, constituting the typical mixed inflammatory cell population. E, Focal area of myositis, where bundles of skeletal muscle fibers are permeated by eosinophils, neutrophils, and plasma cells (H\&E; original magnification $\mathbf{A}, \mathbf{B}, \times 40 ; \mathbf{C}-\mathbf{E}, \times 400)$. A high-resolution version of this slide for use with the Virtual Microscope is available as eSlide: VM02371 and VM02373.

\section{Benign and malignant minor salivary gland tumors} Minor salivary gland tumors represent $10 \%$ to $20 \%$ of all salivary gland tumors. ${ }^{14,15}$ Most lesions occur in the palate, followed by the buccal mucosa, and lips. ${ }^{15}$ Pleomorphic adenoma (PA), mucoepidermoid carcinoma (MEC), and adenoid cystic carcinoma are the most frequent histologic types of intraoral minor salivary gland tumors. ${ }^{14,15}$ In a series of 546 cases of minor salivary gland tumors in a Brazilian population, $305(55.9 \%)$ and $241(44.1 \%)$ were benign and malignant tumors, respectively. This study showed that 33 of 57 benign tumors affecting the buccal mucosa were PAs; and 17 of 41 malignant tumors affecting the buccal mucosa were MECs. ${ }^{15}$ Overall, the highest incidence occurs between the fifth to seventh decades of life, with female gender predilection. ${ }^{15}$ Minor salivary gland tumors usually occur as an asymptomatic, firm and ulcerated, or nonulcerated submucosal mass. ${ }^{14,15}$ Microscopically, PA is composed of both epithelial and myoepithelial elements; MEC presents mucous cells together with epidermoid and intermediate cells, whereas adenoid cystic carcinoma exhibits distinct architectural patterns described as tubular, cribriform, and solid. ${ }^{14,15}$

\section{Benign/reactive and malignant lymphoid proliferations}

Follicular lymphoid hyperplasia is a rare benign lymphoproliferative process, with approximately 20 cases reported in the English language literature to date. ${ }^{16}$ It has been also named benign lymphoid hyperplasia, reactive lymphoid hyperplasia, and pseudolymphoma. Follicular lymphoid hyperplasia occurs most frequently in older female patients (mean age 61 years), usually presenting as a unilateral, painless, and slow-growing mass on the posterior hard palate and occasionally affecting the tongue. Buccal mucosa involvement appears to be rare. Histologically, the lesion is composed of multiple follicles with welldefined germinal centers. ${ }^{16}$

Non-Hodgkin lymphomas (NHLs) represent the third most common group of malignant lesions of the oral 
cavity, just behind squamous cell carcinomas and adenocarcinomas of salivary gland origin. In the oral cavity and oropharynx, NHLs account for $13 \%$ of all primary extranodal NHLs, commonly affecting the tonsils (about 70\%). In the oral cavity, NHLs account for $2 \%$ to $3 \%$ of all malignancies, with most cases representing B-cell lymphomas, followed by $\mathrm{T}$-cell and natural killer/T-cell lymphomas. ${ }^{17,18}$ Oral NHL occurs frequently between the fifth and seventh decades of life, with slight male gender predilection. The palate and tongue are commonly affected, followed by the buccal mucosa, floor of the mouth, and jaws. On clinical examination, oral NHLs may present lesions ranging from swellings to ulcerated masses, which can mimic reactive lesions such as pyogenic granuloma or giant cell lesion, as well as periodontal disease, osteomyelitis, squamous cell carcinoma, and salivary gland neoplasms. $^{17,18}$

\section{Malignant mesenchymal tumors}

Sarcomas are rare malignant tumors of mesenchymal origin, usually classified considering their cell type origin, mainly based on microscopic and immunohistochemical findings, as well as cytogenetic changes. Although about $80 \%$ of sarcomas are derived from mesenchymal cells of soft tissues, a few of them have bony and cartilaginous origin. Soft tissue sarcomas account for about $6 \%$ of all malignant tumors in children and less than $1 \%$ of malignancies among adults. ${ }^{19}$ In the head and neck region, soft tissue sarcomas are uncommon, being their frequency higher in pediatric patients than in adult patients $(35 \%$ vs $10 \%) .{ }^{19}$ Clinically, soft tissue sarcomas of the head and neck region present as an infiltrative mass in the face, neck, sinonasal tract, and oral cavity, usually presenting a rapid onset and involvement of adjacent structures. The most soft tissue sarcomas are fibrosarcoma, leiomyosarcoma, rhabdomyosarcoma, undifferentiated high-grade pleomorphic sarcoma, and synovial sarcoma. ${ }^{19}$ About $20 \%$ of adult patients with head and neck soft tissue sarcoma present distant metastases. Recurrences occur within 2-year after the treatment, and the 5-year survival and local control rates are of $65 \%$ and $70 \%$, respectively. ${ }^{19}$

\section{DIAGNOSIS AND MANAGEMENT}

In the case presented here, by considering the clinical findings and the abrupt onset of the current lesion, an incisional biopsy was performed. The histopathologic evaluation on the hematoxylin and eosin (H\&E) stain revealed a nonulcerated lesion, presenting submucosal diffuse inflammation surrounding minor salivary glands, bundles of skeletal muscle fibers and adipose tissue (Figures 2A-2E). A mixed inflammatory infiltrate consisting of macrophages, plasma cells,
Table I. List of primary antibodies and immunohistochemical results

\begin{tabular}{|c|c|c|c|c|}
\hline Antibody & Dilutions & Clone & Supplier & Results \\
\hline CD1a & $1: 400$ & $\mathrm{O} 10$ & DakoCytomation ${ }^{*}$ & $+1-$ \\
\hline CD3 & $1: 800$ & Polyclonal & DakoCytomation ${ }^{*}$ & ++ \\
\hline CD4 & $1: 200$ & $4 \mathrm{~B} 12$ & Leica Biosystems ${ }^{\dagger}$ & + \\
\hline CD8 & $1: 600$ & C8/144 B & DakoCytomation ${ }^{*}$ & ++ \\
\hline CD11c & $1: 500$ & $5 \mathrm{D} 1$ & Leica Biosystems ${ }^{\dagger}$ & ++ \\
\hline CD20 & $1: 2000$ & L26 & DakoCytomation & $+1-$ \\
\hline CD34 & $1: 100$ & QBEnd/10 & Leica Biosystems ${ }^{\dagger}$ & - \\
\hline CD56 & $1: 100$ & CD564 & Leica Biosystems ${ }^{\dagger}$ & $+1-$ \\
\hline CD57 & $1: 500$ & TB01 & DakoCytomation ${ }^{*}$ & $+/-$ \\
\hline CD68 & $1: 3000$ & KP1 & DakoCytomation* & +++ \\
\hline CD123 & $1: 200$ & BR4 MS & Leica Biosystems ${ }^{\dagger}$ & $+1-$ \\
\hline CD138 & $1: 500$ & MI15 & DakoCytomation ${ }^{*}$ & + \\
\hline CD163 & $1: 500$ & 10D6 & Leica Biosystems & +++ \\
\hline CD207 & $1: 200$ & 12D6 & Monosan $^{\ddagger}$ & $+1-$ \\
\hline CD303 & $1: 500$ & $124 \mathrm{~B} 3.13$ & Dendritics ${ }^{\S}$ & $+1-$ \\
\hline EMA & $1: 500$ & E29 & DakoCytomation ${ }^{*}$ & + \\
\hline Granzyme B & $1: 100$ & GrB-7 & DakoCytomation ${ }^{*}$ & $+I-$ \\
\hline Perforin & $1: 100$ & $5 \mathrm{~B} 10$ & Leica Biosystems ${ }^{\dagger}$ & - \\
\hline HLA-DR & $1: 500$ & TAL.1 B5 & DakoCytomation ${ }^{*}$ & +++ \\
\hline S100 & $1: 3000$ & Polyclonal & Leica Biosystems ${ }^{\dagger}$ & + \\
\hline XIIIIa & $1: 100$ & E980.1 & Leica Biosystems ${ }^{\dagger}$ & + \\
\hline Ki-67 & $1: 500$ & MIB-1 & DakoCytomation ${ }^{*}$ & $>7 \%$ \\
\hline
\end{tabular}

- , negative; $+/-$, rare positive cells;,$+ \leq 5 \%$ of positivity; ++ , $5 \%-50 \% ;+++,>50 \%$.

*Glostrup, Denmark.

${ }^{\dagger}$ Newcastle, UK.

${ }^{\ddagger}$ Uden, Netherlands.

${ }^{\S}$ Lyon, France.

lymphocytes, neutrophils and eosinophils, which involved the residual salivary gland parenchyma, with foci of acinar atrophy and necrosis, as well as myositis areas and remarkably nongranulomatous macrophage-rich sheets, were evident. Initially, the inflammatory process raised the suspicion of an infectious process. However, special histochemical stains, such as periodic acid-Schiff (PAS), Grocott-Gomori methenamine silver, acid-fast bacilli and Gram, were all negative. Thus, the clinicopathologic findings were consistent with the diagnosis of subacute necrotizing sialadenitis (SANS). The lesion healed completely after 4 weeks of follow-up (Figure 1B).

Immunohistochemical analysis was performed to characterize and highlight the heterogeneous population of the inflammatory cells, through a broad panel of antibodies, such as shown in the Table I. The percentage of positive cells was determined by using the image computer analyzer (Dako's Automated Cellular Imaging System; Dako, Carpinteria, CA). Two authors (J.E.L. and L.Y.A.) performed blinded analysis of randomly immunohistochemical slides independently. It was classified as - , negative; $+/-$, rare positive cells;,$+ \leq 5 \%$ of positivity;,$++ 5 \%$ to $50 \%$ of positivity;,$+++>50 \%$ of positivity. The results showed positivity for $\mathrm{CD} 3$ and $\mathrm{CD} 8$, followed by 

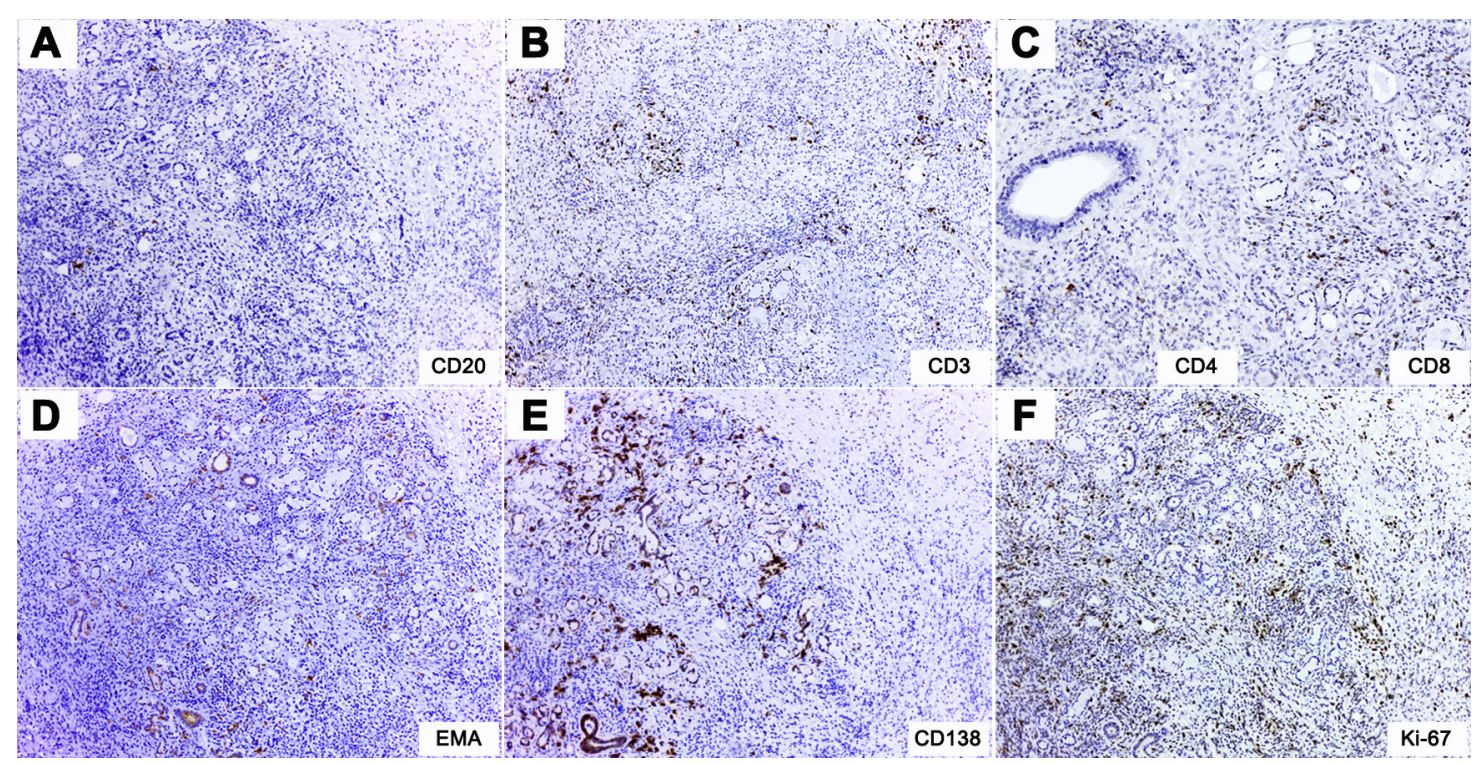

Fig. 3. Immunohistochemical findings. A, Few CD20+ cells. B, Numerous CD3+ cells. C, A higher number of CD $8+$ cells, rather than CD4+ cells, was observed. Immunoexpression of EMA (D) and CD138 (E) highlighting residual parenchyma of minor salivary glands, as well as scarce plasma cells. F, Ki-67 shows positivity in numerous inflammatory cells (7\%) (original magnification A, B, D-F, $\times 100 ; \mathbf{C}, \times 200)$.
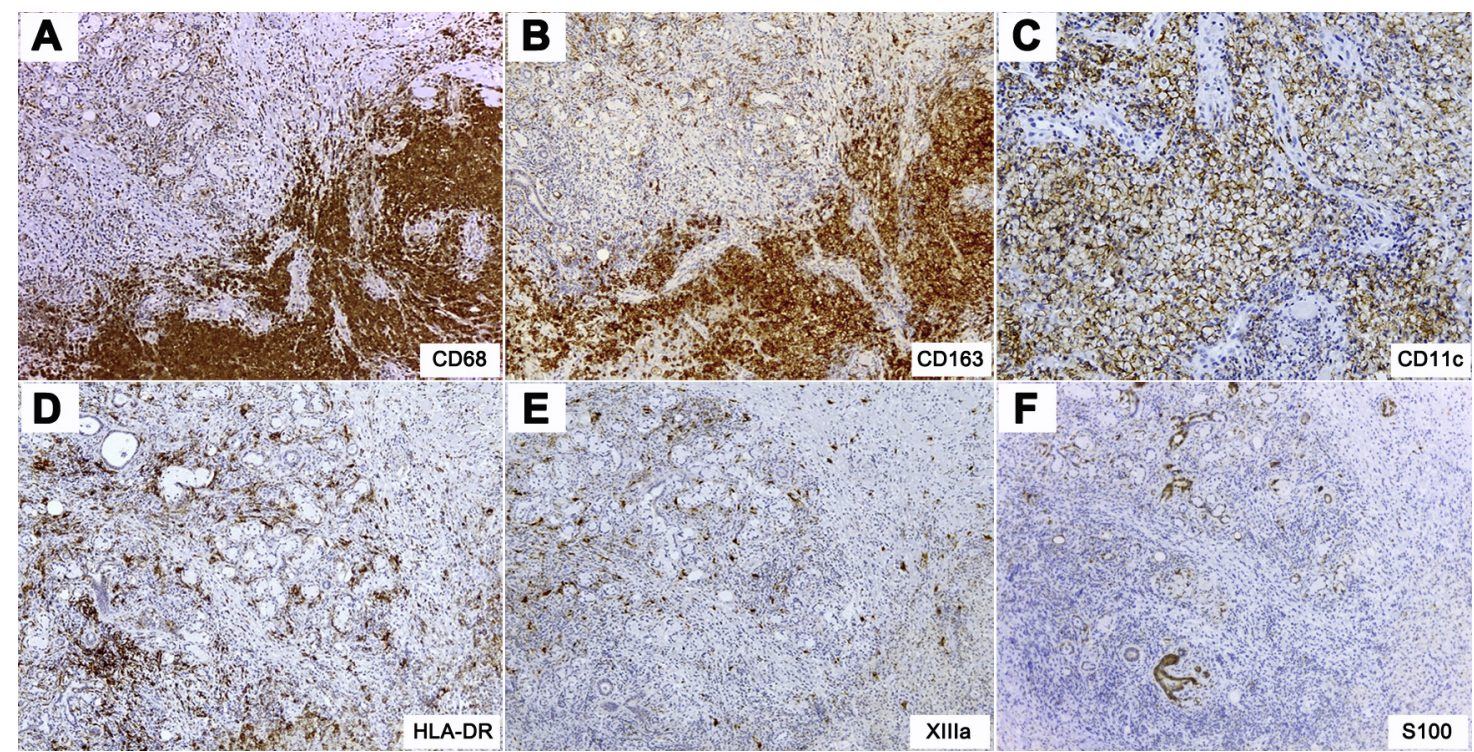

Fig. 4. Immunohistochemical findings. Through consecutive serial sections, strong immunoexpression for CD68 (A), CD163 (B) and CD11c (C) suggested a M2-like phenotype for macrophage population. D, HLA-DR highlighted numerous dendritic-like cells. E, Diffuse pattern of XIIIa positive cells, suggesting a submucosal dendritic cell population. F, S100 highlighted the parenchyma of minor salivary glands and scarce inflammatory cells with dendritic morphology (original magnification $\mathbf{A}, \mathbf{B}, \mathbf{D}-\mathbf{F}, \times 100$; C, $\times 200)$.

CD4, in diffuse pattern, whereas CD20+ B-cells were practically absent. These data suggest a predominance of cytotoxic T-cells (CD3+/CD8+) (Figures 3A-3C). EMA and CD138 immunostaining revealed focal aggregates of plasma cells; moreover, these markers highlighted the residual salivary gland component (Figures 3D and 3E). In consecutive serial sections, macrophages were strongly immunopositive for both CD68 and CD163, suggesting a M2-like phenotype (Figures 4A and 4B). This finding was also confirmed by $\mathrm{CD} 11 \mathrm{c}$ positivity, a macrophage marker (Figure 4C). Human leukocyte antigen-D related (HLA-DR) highlighted, in addition to macrophages, a significant number of dendritic-like cells (Figure 4D). 
Among these latter, submucosal dendritic cells were evidenced by XIIIa immunomarker (Figure 4E). Scarce $\mathrm{S} 100+$ dendritic-like cells were visualized (Figure 4F), whereas CD1a+/CD207+ Langerhans cells were practically absent. Interestingly, S100 positivity was also detected in some ductal cells and seromucous acini of the residual salivary glands. Furthermore, plasmacytoid dendritic cells (CD123+/ CD303+) were occasionally seen. CD56, CD57, and granzyme $\mathrm{B}$ confirmed the presence of scattered cytotoxic lymphocytes. CD34 highlighted discrete fine blood vessels within the lesional stroma. Last, inflammatory cells expressing Ki-67 proliferation marker were meaningful $(>7 \%)$, especially on macrophage and lymphocyte populations (Figure $3 \mathrm{~F}$ ).

\section{DISCUSSION}

SANS was first described in 1990, as a self-limiting inflammatory process. The etiology of SANS is unknown, but traumatic, infectious, and allergic etiologies have been suggested. ${ }^{20-22}$ Including the current case, only 33 SANS cases have been reported in the English language literature. Of them, 21 occurred on the hard palate, four on the soft palate, four on the buccal mucosa, two on the tongue, one on the upper lip, and one on the tonsillar area. ${ }^{20-22}$ Interestingly, none of them was found affecting the major salivary glands. The majority of cases occurred between the second and third decades of life (mean age 26.7 years), affecting preferentially men (ratio $3: 1) .^{20-22}$ Commonly, this lesion presents as a localized nonulcerated painful swelling, usually not exceeding $2 \mathrm{~cm}$ in diameter. ${ }^{22}$ As previously mentioned, in four SANS cases, the buccal mucosa was affected, in three women and one man, with an age range of 30 to 78 years (mean age 57.2 years). Histologically, SANS is characterized by a diffuse infiltration of both acute and chronic inflammatory cells involving the parenchyma of minor salivary glands, which show ductal atrophy and acinar necrosis. ${ }^{21}$ Ductal squamous metaplasia is mild or absent. Interestingly, eosinophilia is a constant finding among the most reported cases of SANS, ${ }^{22}$ and it was also evidenced in the current case (Figures 2C-2E). Eosinophils are usually absent in necrotizing sialometaplasia (NS) and chronic sclerosing sialadenitis (CSS), which are the main differential diagnoses of SANS. $^{22}$ Despite some authors consider SANS as unusual subtype of NS, ${ }^{20-22}$ there are clinical and histologic features that suggest it to be a distinct entity. The major ones among them are that SANS presents as a nonulcerated swelling, which shows a subacute inflammatory infiltrate and the average duration before diagnosis is less than 1 week. In contrast, NS is characterized by an ulcerated swelling composed of chronic inflammatory infiltrate and squamous metaplasia, which has a duration of approximately 3 weeks. ${ }^{21,22}$ Moreover, different from SANS, NS cases are most frequently diagnosed in other locations, such as major salivary glands, nasal cavity, maxillary sinus, and larynx. ${ }^{21}$

By analysis of the H\&E staining, the current SANS case exhibited numerous macrophages, neutrophils, eosinophils, plasma cells, and lymphocytes. To the best of our knowledge, this is the first study that provided a detailed immunohistochemical profile of the inflammatory cell populations in SANS. Overall, the inflammatory infiltrate consisted predominantly of M2-like phenotype macrophages, followed by dendritic cells (myeloid origin) and cytotoxic T-lymphocytes. The higher number of CD3+ T-cells, associated with the practically absence of CD20 + B cells, suggested that SANS has a predominant cell-mediated immune response. Macrophages are capable of responding to environmental changes by altering their functional phenotype, depending on surrounding signals. M1 macrophages (CD68+/CD163-/HLA-DR+) are proinflammatory, and they are characterized by high levels of phagocytosis, inhibition of cell proliferation, and tissue damage. ${ }^{23}$ In the current case, it was observed that the vast majority of macrophages exhibited the M2-like phenotype (CD68+/CD163+/HLA-DR+), which is anti-inflammatory and responsible for the angiogenesis and the tissue repair. ${ }^{23}$ Finally, by considering the dendritic cell populations, this study found that the myeloid lineage was predominant over plasmacytoid lineage. In fact, CD123 and CD303 immunomarkers exhibited scarce positive cells. Myeloid dendritic cells play a major role in antigen presentation and are capable of induce T-cell immunity or tolerance, depending on their stage of maturation. ${ }^{24}$ In contrast, plasmacytoid dendritic cells produce a massive amount of interferon type I upon viral infection and have an immunomodulatory role in natural killer, T-cell, and B-cell responses. ${ }^{24}$ Therefore, our results suggest the participation of dendritic cell subsets in the pathogenesis of SANS. Future studies, including a large SANS series, are necessary for a better defining of their etiopathogenic mechanisms and its delineation from others reactive conditions affecting minor salivary glands, such as NS.

The authors thank the Pathology Digital Technologies Core (NTDP) at Pathology Department at Botucatu Medical School, UNESP, for technical support with digital whole slides.

\section{REFERENCES}

1. Chrysomali E, Papanicolau SI, Dekker NP, Regezi JA. Benign neural tumors of the oral cavity: a comparative immunohistochemical study. Oral Surg Oral Med Oral Pathol Oral Radiol Endod. 1997;84:381-390. 
2. Lerman MA, Li CC, Woo SB. Dendritic cell neurofibroma with pseudorosettes: a clinicopathologic and immunohistochemical study of 5 intraoral cases. Oral Surg Oral Med Oral Pathol Oral Radiol. 2014;117:221-226.

3. Studart-Soares EC, Costa FW, Sousa FB, Alves AP, Osterne RL. Oral lipomas in a Brazilian population: a 10-year study and analysis of 450 cases reported in the literature. Med Oral Patol Oral Cir Bucal. 2010;15:e691-e696.

4. Manor E, Sion-Vardy N, Joshua BZ, Bodner L. Oral lipoma: analysis of 58 new cases and review of the literature. Ann Diagn Pathol. 2011;15:257-261.

5. O'Regan EM, Vanguri V, Allen CM, Eversole LR, Wright JM, Woo SB. Solitary fibrous tumor of the oral cavity: clinicopathologic and immunohistochemical study of 21 cases. Head Neck Pathol. 2009;3:106-115.

6. Eley KA, Alroyayamina S, Golding SJ, Tiam RN, WattSmith SR. Angioleiomyoma of the hard palate: report of a case and review of the literature and magnetic resonance imaging findings of this rare entity. Oral Surg Oral Med Oral Pathol Oral Radiol. 2012;114:e45-e49.

7. Veeresh M, Sudhakara M, Girish G, Naik C. Leiomyoma: a rare tumor in the head and neck and oral cavity: Report of 3 cases with review. J Oral Maxillofac Pathol. 2013;17:281-287.

8. Vered M, Allon I, Buchner A, Dayan D. Clinico-pathologic correlations of myofibroblastic tumors of the oral cavity. II. Myofibroma and myofibromatosis of the oral soft tissues. J Oral Pathol Med. 2007;36:304-314.

9. Azevedo Rde S, Pires FR, Della Coletta R, de Almeida OP, Kowalski LP, Lopes MA. Oral myofibromas: report of two cases and review of clinical and histopathologic differential diagnosis. Oral Surg Oral Med Oral Pathol Oral Radiol Endod. 2008;105: 35-40.

10. de Feraudy S, Fletcher CD. Intradermal nodular fasciitis: a rare lesion analyzed in a series of 24 cases. Am J Surg Pathol. 2010;34:1377-1381

11. Lloyd AA, Witheiler D, Menter A. Nodular fasciitis of the lip mucosa: a rare but clinically important entity. Clin Exp Dermatol. 2015;40:408-412.

12. Piña AR, Almeida LY, Andrade BA, León JE. Clear cell change in a lower lip mucocele. J Oral Maxillofac Pathol. 2013;17:318.

13. Rangel AL, León JE, Jorge J, Lopes MA, Vargas PA. Oncocytic metaplasia in inflammatory fibrous hyperplasia: histopathological and immunohistochemical analysis. Med Oral Patol Oral Cir Bucal. 2008;13:E151-E155.

14. Eveson JW, Cawson RA. Salivary gland tumours. A review of 2410 cases with particular reference to histological types, site, age and sex distribution. J Pathol. 1985;146:51-58.
15. Pires FR, Pringle GA, de Almeida OP, Chen SY. Intra-oral minor salivary gland tumors: a clinicopathological study of 546 cases. Oral Oncol. 2007;43:463-470.

16. Anjomshoaa I, Bulford LA, Dym H, Woo SB. Florid follicular lymphoid hyperplasia of the hard palatal mucosa managed with intralesional steroids: a case report and review of the literature. J Oral Maxillofac Surg. 2013;71:1202-1208.

17. Epstein JB, Epstein JD, Le ND, Gorsky M. Characteristics of oral and paraoral malignant lymphoma: a population-based review of 361 cases. Oral Surg Oral Med Oral Pathol Oral Radiol Endod. 2001;92:519-525.

18. Carlos Bregni R, Nuyens M, Vassallo J, et al. Marginal zone lymphoma of mucosa-associated lymphoid tissue with prominent plasma cell differentiation affecting the palatine tonsil: histopathological and immunohistochemical analysis. Oral Surg Oral Med Oral Pathol Oral Radiol. 2012;113:526-532.

19. Romañach MJ, León JE, de Almeida OP, Carlos R. Soft tissue sarcomas of the head and neck. SADJ. 2012;67:582-584.

20. Werning JT, Waterhouse JP, Mooney JW. Subacute necrotizing sialadenitis. Oral Surg Oral Med Oral Pathol. 1990;70(6): 756-759.

21. Fowler CB, Brannon RB. Subacute necrotizing sialadenitis: report of 7 cases and a review of the literature. Oral Surg Oral Med Oral Pathol Oral Radiol Endod. 2000;89:600-609.

22. Suresh L, Aguirre A. Subacute necrotizing sialadenitis: a clinicopathological study. Oral Surg Oral Med Oral Pathol Oral Radiol Endod. 2007;104:385-390.

23. Mills CD. M1 and M2 macrophages: oracles of health and disease. Crit Rev Immunol. 2012;32:463-488.

24. Steinbrink K, Mahnke K, Grabbe S, Enk AH, Jonuleit H. Myeloid dendritic cell: from sentinel of immunity to key player of peripheral tolerance? Hum Immunol. 2009;70:289-293.

\section{Reprint requests:}

Luciana Yamamoto Almeida

Department of Diagnosis and Surgery

Araraquara Dental School

Universidade Estadual Paulista (UNESP)

Rua Humaitá, n. 1680

CEP. 14801-903

Araraquara

Sao Paulo

Brazil

al.yamamoto@hotmail.com 